\title{
Hybrid Molecular Imprinted Polymer (MIP) Electrode for Pesticide Detection of Electrochemical Sensor
}

\author{
Abdul Latif Ahmad, Nuur Fahanis Che Lah* and Low Siew Chun \\ School of Chemical Engineering, Engineering Campus, \\ Universiti Sains Malaysia, 14300 Nibong Tebal, \\ Seberang Perai Selatan, Pulau Pinang, Malaysia \\ *Corresponding author: fahanischelah87@gmail.com
}

Published online: 25 February 2018

To cite this article: Ahmad, A. L., Che Lah, N. F. \& Low, S. C. (2018). Hybrid molecular imprinted polymer (MIP) electrode for pesticide detection of electrochemical sensor. J. Phys. Sci., 29(Supp. 1), 33-40, https://doi.org/10.21315/jps2018.29.s1.5

To link to this article: https://doi.org/10.21315/jps2018.29.s1.5

\begin{abstract}
Molecular imprinting technology is becoming a versatile tool for the preparation of tailor-made molecular recognition elements. An innovative electrochemical sensor which enables the recognition of a small pesticide target molecule atrazine based on molecularly imprinted polymer (MIP) is developed. The MIP has been synthesised by using bulk polymerisation method. Further attachment of the polymer onto the graphite felt activated the MIP-electrode for electrochemical sensor. The obtained sensing MIP is highly specific towards newly added atrazine and the recognition can be quantitatively analysed by the variation of the cyclic voltammogram of hybrid MIP-electrode. The developed sensor shows remarkable properties with 1.2 of imprinting factor with binding capacity of $4.06 \pm 0.8 \mathrm{mg} \mathrm{g}^{-1}$ of polymer.
\end{abstract}

Keywords: Molecular imprinted polymer, pesticide, electrochemical, biosensor, pesticide detection

\section{INTRODUCTION}

The disposal of pesticide-containing waste is a problem of worldwide concern, with almost every stage of pesticide use involving the formation of waste, which can contain substances that are strictly controlled by regulatory bodies. The presence of large stocks of pesticides that are unusable, either due to the fact that they are banned or have exceeded their self-life, is of great concern especially in developing countries. The geographic distribution of pesticide concentrations generally follows regional patterns in agricultural use and the influence of urban areas, although this 
relation is stronger for streams than for groundwater. Compared with streams, the occurrence of pesticides in groundwater is more strongly governed by compound properties and hydrogeologic factors that affect transport from land surface to a well. ${ }^{1}$

An increasing effort is now being put into the environmental monitoring of pesticides that may pose a risk to human and ecosystem health. Earlier techniques used for pesticide detection were chromatographic methods like gas chromatography (GC), high performance liquid chromatography (HPLC) along with mass spectrometry (MS), etc. They were sensitive and reliable. ${ }^{2}$ The time and expenses involved in classical analytical methods (i.e., sampling, sample preparation, and laboratory analysis) limit the number of samples that can be analysed in environmental surveys. There is a real need for developing fast, easy-to-use, robust, sensitive, cost-effective and field-analytical techniques. ${ }^{3}$

Detection of pesticides at the levels established by the Environmental Protection Agency (EPA) remains a challenge. Chromatographic methods coupled with selective detectors have been traditionally used for pesticide analysis due to their sensitivity, reliability and efficiency. Nevertheless, they are time consuming and laborious, and require expensive equipment and highly trained technicians. Over the past decade, considerable attention has been given to the development of biosensors for the detection of pesticides as a promising alternative. A biosensor is a self-contained device that integrates an immobilised biological element (e.g., enzyme, DNA probe, antibody) that recognises the analyte (e.g., enzyme sub-strate, complementary DNA, antigen) and a transduction element used to convert the (bio)chemical signal resulting from the interaction of the analyte with the bioreceptor into an electronic one. According to the signal transduction technique, biosensors are classified into electrochemical, optical, piezoelectric and mechanical biosensors. ${ }^{4}$

The integration of molecular imprinting and electrochemical has produced high selectivity and sensitivity sensor which use molecularly imprinted polymers (MIPs) as specific recognition element for template molecule detection. This hybrid technology may present considerable potential for the development of devices that offer significant advantages compared to the current methodologies. Up to now, these hybrid technologies have been successfully used to detect many different kinds of molecules, including of pharmaceuticals, metabolites, protein and so on. Recently, many kinds of surface-imprinted polymers have been reported.

Several studies have been conducted regarding the detection of atrazine by using molecular imprinted polymers. Pardieu et al. have built an electrochemical sensor for this purpose. The conjugated molecular imprinted conducting polymer 
(MICP) has been electrochemically synthesised onto a platinum electrode. ${ }^{5}$ The obtained MICP is highly specific towards newly added atrazine with selectivity of detection between $10^{-9} \mathrm{~mol} \mathrm{l}^{-1}$ to $1.5 \times 10^{-2} \mathrm{~mol}^{-1}$ and low threshold $\left(10^{-7} \mathrm{~mol}^{-1}\right)$. Chen et al. presented the work of imprinted polymers by far-infrared and ultraviolet induced polymerisation. ${ }^{6}$

This paper presents the study of the prospective of electrochemical sensor for atrazine using a commercial graphite felt electrode hybrid with molecular imprinted polymer. The effect of using different shot of binder, varying the electrolyte concentration and different scan rate are investigated. The results are also compared with those obtained for other electrodes.

\section{EXPERIMENTAL}

The protocol for MIP synthesis was modified from Kueseng et al. ${ }^{7}$ The atrazineimprinted polymer was prepared by dissolving template (atrazine, Atr) in the porogen (toluene, $0.7 \mathrm{ml}$ ) in a $7 \mathrm{ml}$ glass tube. Functional monomer (methacrlic acid, MAA), crosslinker (ethylene glycol dimethacrylate, EGDMA) and initiator (azobisisobutyronitrile: AIBN) were then added to the mixture and purged with nitrogen for $5 \mathrm{~min}$. The tube was sealed and placed in a water bath at $60^{\circ} \mathrm{C}$ for $20 \mathrm{~h}$. As a control, non-imprinted polymer (NIP) was simultaneously prepared in the same manner but without the addition of the template. The bulk polymer obtained was smashed, ground and dried in a desiccator for $24 \mathrm{~h}$.

The rebinding properties of MIP and NIP for atrazine were studied. An amount of $5 \mathrm{ml}$ of atrazine solution at an initial concentration of $20 \mathrm{ppm}$ was added to 25 $\mathrm{mg}$ of polymer (MIP or NIP). The mixture was left for 120 min-adsorption under constant shaking at room temperature $\left(30^{\circ} \mathrm{C}\right)$. the amount of atrazine adsorbed by MIP and NIP was determined by HPLC (Agilent Technologies, USA) by injecting $10 \mu 1$ of each analyte to a $\mathrm{C} 18$ analytical column which was controlled at $40^{\circ} \mathrm{C}$. Atrazine was quantified at wavelength of $230 \mathrm{~nm}$ with an effluent of methanol and water ratio of 55:45 at a flow rate of $0.4 \mathrm{ml} \mathrm{min}{ }^{-1}$. All injections were repeated at least three times and the average value was reported with the standard deviation. The adsorption capacity, Q ( $\mathrm{mg} \mathrm{g}^{-1}$ of polymer) of atrazine bound to the polymer was calculated by the following equation:

$$
\mathrm{Q}=\frac{\left(C_{0}-C_{t}\right) V}{W}
$$

where $\mathrm{C}_{0}$ and $\mathrm{C}_{\mathrm{t}}\left(\mathrm{mg} \mathrm{l}^{-1}\right)$ are the initial and residual atrazine concentrations at time $\mathrm{t}$, respectively. $\mathrm{V}(\mathrm{l})$ is the volume of atrazine solution and $\mathrm{W}(\mathrm{g})$ stands the mass of MIP and NIP. 
The raw graphite felt used in this study defining an open framework with cavities of ca. $100 \mu \mathrm{m}$. The graphite felt was first dipped in ethanol to eliminate air bubbles. For prior electrochemistry experiments, the felt was rinsed in deionised water and dried for 12 h. $20 \%$ PVC in NMP coated the graphite felt as the binder to the imprinted polymer. The cyclic voltammetric measurements were performed by using VMP3 potentiostat. The electrochemical setup used in this study was based on three electrode system. Calomel Reference Electrode as the reference electrode and Pt Counter Electrode as the counter electrode was chosen in this setup. All the experiments were carried out at range of $-2.00 \mathrm{~V}$ and $2.00 \mathrm{~V}$ versus reference electrode with scanning rate of $50 \mathrm{mV} \mathrm{s}^{-1}$. The SEM was conducted at magnification of 1000 .

\section{RESULTS AND DISCUSSION}

Tailoring of a functional monomer to the template molecules is the most important step in molecular imprinting process. A common used monomer, MAA was tested in the MIPs preparation. MAA displayed the best effectiveness as functional monomer when used to imprinting atrazine because they can form double hydrogen bond with atrazine, which leads to an understanding that a functional monomer is required to have both strong binding and multiple binding capacities. ${ }^{8}$ The resulting shape of particles was like irregular MIPs aggregates. Through the binding interactions, the adsorption capacity of Atrazine-MIP synthesised in the present work could be dominated by: (1) the shape factor of the recognition cavities that is able to "lock" the target atrazine in position; and (2) the availability of the imprinted specific recognition sites that attracted atrazine.

As the MAA was chosen as the monomer for the preparation of atrazine imprinted polymer, its suitable dosage was pivotal to determine the formation of optimum imprinted sites. Keeping the molar ratio of template:cross-linker (Atr:EGDMA) constant at 1:25, the adsorption profiles of atrazine by MIP and NIP were evaluated at different molarities of MAA, as depicted in Figure 1. The adsorption experiments were triplicated. As indicated by Figure 1, a tremendous decrement of binding capacity was illustrated when the molarity of MAA was altered from 5 to 30 . MIP unraveled poor binding at high molarities of MAA, i.e., at 30, the amount of atrazine adsorbed was $2.14 \pm 0.36 \mathrm{mg} \mathrm{g}^{-1}$ of MIP. It could be that the higher monomer concentration leads to bulk monolith. ${ }^{9}$

Moreover, the similar trend of imprinting factor can be observed from Figure 1. As the monomer concentration increased, the imprinting factor is decreased until it reached some point that there is no imprinting at all (IF below than one). The dilute medium can adversely affect the template monomer complexation, shifting 
its equilibrium towards the uncomplexed forms hence the number of imprinted binding sites might significantly decrease. The highest binding capacity sample (monomer ratio: 5) was selected for further study in construction of electrochemical sensor.

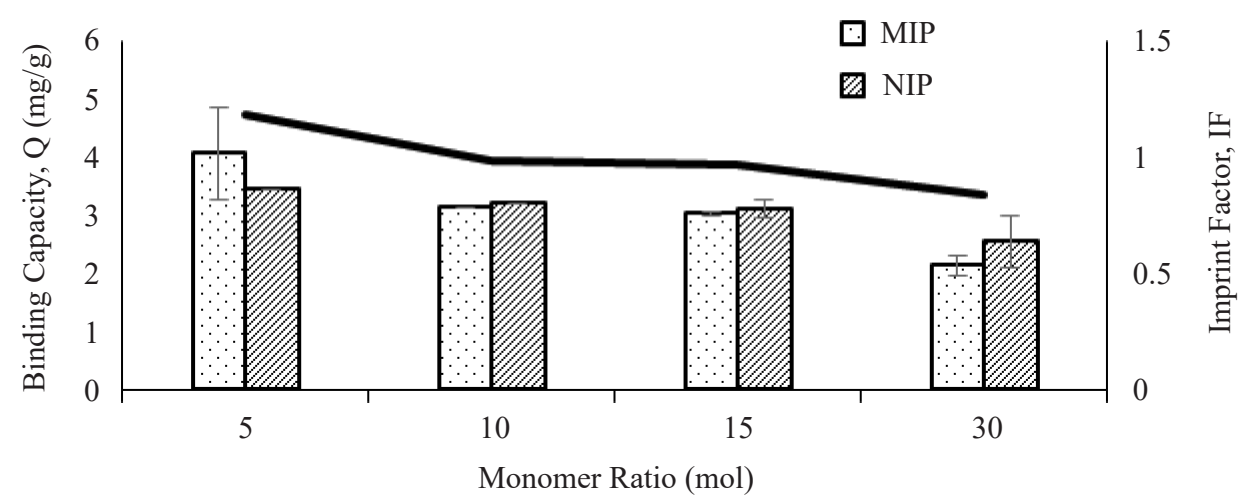

Figure 1: Absorbed amounts of atrazine by MIP and NIP with imprinting factor for different monomer concentration.

Note: Polymer synthesised using a constant molar ratio of template:cross linker at 1:25 (MIP) and $0: 25$ (NIP).

Experiments were first carried out to check the electrochemical behaviour of atrazine on a graphite felt electrode in the absence of MIP. This electrochemical study was performed by cyclic voltammetry at a scanning rate of $50 \mathrm{mV} \mathrm{s}^{-1}$. Figure 2 displays the cathodic currents measured at graphite felt electrode in the absence of pesticides (line a) and for $20 \mathrm{ppm}$ concentration of atrazine (line b). In cyclic voltammograms obtained in the presence of atrazine, reduction peaks can be observed while the oxidation is embedded in a large wave with no clear oxidation peak. This observation is consistent with previous studies concerning atrazine reduction on graphite felt electrode and implies that atrazine reduction, which is estimated to be a two-electron process, is an irreversible process. ${ }^{10}$ The above electrochemical study of atrazine allows now to understand the redox behaviour of molecular imprinted polymers on graphite felt electrode.

Assays were performed to evaluate the behaviour of the modified electrode during atrazine detection. Figure 3 shows the electrochemical behaviour for imprinted and non-imprinted polymer in $20 \mathrm{ppm}$ of atrazine solution with $0.1 \mathrm{M} \mathrm{NaCl}$. This figure concluded that the imprinted polymer gives higher reduction conductivity compared to non-imprinted polymer. The low signal of NIP electrode can be attributed to the lack of molecular recognition since NIP does not possess the cavities that would provide selective active sites. ${ }^{11}$ MIP electrode provides good reduction currents, indicating that the cavities do present even after binding the 
polymer to the electrode with PVC. The fact that only MIPs are able to interact, and then to detect atrazine, demonstrates the essential presence of pre-shaped cavities into the opolymer matrix. Lower detection of NIPs indicates that the unspecific adsorption of target molecules as well as their interaction by " $\pi$ - $\pi$ stacking" with aromatic groups of the copolymers can be neglected for both NIPs and MIPs. ${ }^{10}$

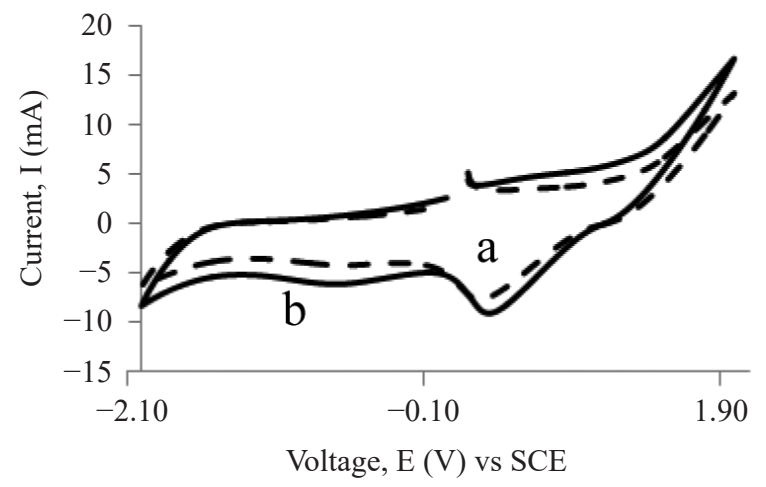

Figure 2: Cyclic voltammograms at scanning rate $50 \mathrm{mV} \mathrm{s}^{-1}$ for bare electrode in (a) $0.1 \mathrm{M} \mathrm{NaCl}$ solution, and (b) $0.1 \mathrm{M} \mathrm{NaCl}$ with $20 \mathrm{ppm}$ atrazine solution.

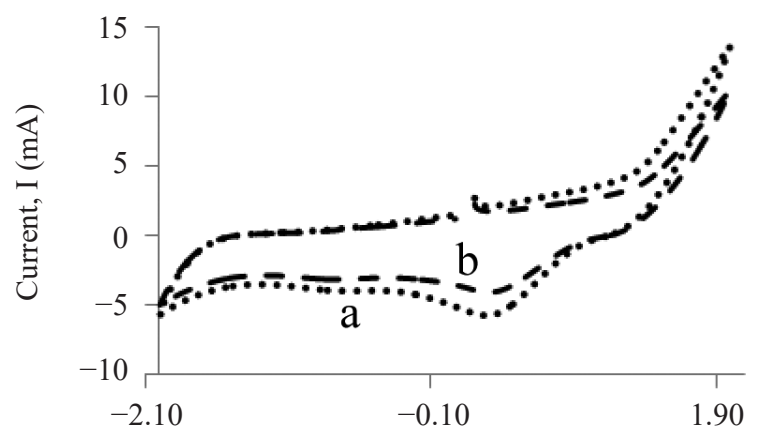

Voltage, E (V) vs SCE

Figure 3: Cyclic voltammograms at scanning rate $100 \mathrm{mV} \mathrm{s}^{-1}$ for initial atrazine concentration of $20 \mathrm{ppm}$ for (a) MIP (b) NIP.

SEM images of the graphite felt are shown in Figure 4. The biopolymer appears to form large aggregates between the carbon fibres. Indeed these images are obtained after drying of the felt so it is difficult to infer alginate organisation in the wet state. Nevertheless, it is important to point out that the PVC coating does not exert a significant influence on the porous structure of the felt. Accordingly, surface modification did not impact the conduction properties of the carbon network. 

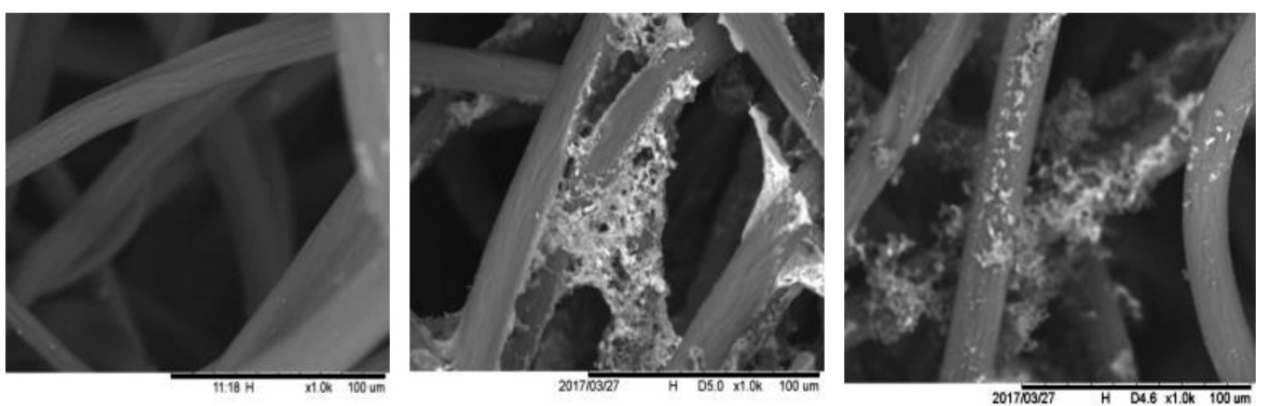

Figure 4: SEM images for bare electrode (left), electrode-polymer surface (middle), and electrode-polymer cross-section image (right).

\section{CONCLUSION}

In conclusion, the MIPs embedded on the graphite felt electrode constitute an electrochemical sensor able to detect additional atrazine molecules owing to the presence of preformed functionalise cavities bearing the spatial, conformational and molecular memories of the target.

\section{ACKNOWLEDGEMENTS}

The authors acknowledge the financial support provided by Universiti Sains Malaysia (USM) and RU Membrane Science and Technology Cluster. Also to the Ministry of Higher Education (MOHE) Malaysia for Fundamental Research Grant Scheme (FRGS) (Grant no: 203/PJKIMIA/6071249) and Long Term Research Grant Scheme (LRGS) (Grant no: 203/PJKIMIA/6727101).

\section{REFERENCES}

1. Gilliom, R. J. et al. (1999). Peer reviewed: Testing water quality for pesticide pollution. Environ. Sci. Technol., 33(7), 164A-169A, https://doi.org/ 10.1021/es992770k.

2. Bhadekar, R. et al. (2011). Developments in analytical methods for detection of pesticides in environmental samples. Am. J. Anal. Chem., 2(8A), 1-15, https://doi.org/10.4236/ajac.2011.228118.

3. Hennion, M. C. \& Barcelo, D. (1998). Strengths and limitations of immunoassays for effective and efficient use for pesticide analysis in water samples: A review. Anal. Chim. Acta, 362(1), 3-34, https://doi.org/10.1016/ S0003-2670(97)00608-9. 
4. Audrey Sassolas, B. P-S. \& Marty, J-L. (2012). Biosensors for pesticide detection: New trends. Am. J. Anal. Chem., 3(3), 210-232, https://doi.org/ 10.4236/ajac.2012.33030.

5. Pardieu, E. et al. (2009). Molecularly imprinted conducting polymer based electrochemical sensor for detection of atrazine. Anal. Chim. Acta, 649(2), 236-245, https://doi.org/10.1016/j.aca.2009.07.029.

6. Chen, J. et al. (2014). Atrazine molecular imprinted polymers: Comparative analysis by far-infrared and ultraviolet induced polymerization. Int. J. Mol. Sci., 15(1), 574-587, https://doi.org/10.3390/ijms15010574.

7. Kueseng, P. et al. (2009). Molecularly imprinted polymer for analysis of trace atrazine herbicide in water. J. Environ. Sci. Health B, 44(8), 772-780, https://doi.org/10.1080/03601230903238319.

8. Xu, S., Li, J. \& Chen, L. (2011). Molecularly imprinted polymers by reversible addition-fragmentation chain transfer precipitation polymerization for preconcentration of atrazine in food matrices. Talanta, 85(1), 282-289, https://doi.org/10.1016/j.talanta.2011.03.060.

9. Renkecz, T., László, K. \& Horváth, V. (2014). Molecularly imprinted microspheres prepared by precipitation polymerization at high monomer concentrations. Mol. Impr., 2(1), 1-17, https://doi.org/10.2478/molim2014-0001.

10. Pardieu, E. et al. (2009). Molecularly imprinted conducting polymer based electrochemical sensor for detection of atrazine. Anal. Chim. Acta, 649(2), 236-245, https://doi.org/10.1016/j.aca.2009.07.029.

11. Neto, J. R. M. et al. (2011). A hemin-based molecularly imprinted polymer (MIP) grafted onto a glassy carbon electrode as a selective sensor for 4-aminophenol amperometric. Sens. Actuat. B. Chem., 152(2), 220-225, https://doi.org/10.1016/j.snb.2010.12.010. 